\title{
Concentração de proteínas no humor aquoso de pacientes com g'laucoma primário de ângulo aberto em tratamento clínico
}

\author{
Aqueous humor protein concentration in patients with primaryopen-angle \\ glaucoma underclinical treatment
}

\author{
Tiago dos Santos Prata ${ }^{1}$ \\ Eduardo Vitor Navajas ${ }^{2}$ \\ Luiz Alberto Soares Melo Jr. ${ }^{3}$ \\ João Roberto Maciel Martins ${ }^{4}$ \\ Helena Bonciani Nader ${ }^{5}$ \\ Rubens Belfort Jr. ${ }^{6}$
}

Trabalho realizado no Departamento de Oftalmologia da Universidade Federal de São Paulo.

${ }^{1}$ Preceptor da Residência Médica do Departamento de Oftalmologia da Universidade Federal de São Paulo UNIFESP - São Paulo (SP) - Brasil.

${ }^{2}$ Pós-Graduando do Departamento de Oftalmologia da UNIFESP - São Paulo (SP) - Brasil.

${ }^{3}$ Pós-Graduando do Departamento de Oftalmologia da UNIFESP - São Paulo (SP) - Brasil.

${ }^{4}$ Médico da Disciplina de Endocrinologia do Departamento de Medicina e da Disciplina de Biologia Molecular - Departamento de Bioquímica da UNIFESP - São Paulo (SP) - Brasil.

${ }_{5}^{5}$ Professora Titular da Disciplina de Biologia Molecular do Departamento de Bioquímica da UNIFESP - São Paulo (SP) - Brasil.

${ }^{6}$ Professor Titular do Departamento de Oftalmologia da UNIFESP - São Paulo (SP) - Brasil.

Endereço para correspondência: Tiago dos Santos Prata. Al. Campinas, 1232 - Apto. 71 - São Paulo (SP) CEP 01404-001

E-mail: tiagoprata@oftalmo.epm.br

Recebido para publicação em 01.04.2006

Última versão recebida em 19.09.2006

Aprovação em 16.10.2006

\section{RESUMO}

Objetivos: Comparar a concentração total de proteínas no humor aquoso entre pacientes com glaucoma primário de ângulo aberto e sem glaucoma. Métodos: Foram coletadas amostras de humor aquoso de 22 pacientes com glaucoma primário de ângulo aberto (grupo GPAA) no momento da trabeculectomia. Na coleta, $0,1 \mathrm{~mL}$ de humor aquoso foi aspirado da câmara anterior através de uma agulha de calibre 26, no início do procedimento cirúrgico. Coleta semelhante foi realizada em 22 pacientes sem glaucoma no início da cirurgia de catarata (grupo controle). A amostra de humor aquoso foi armazenada a $-20^{\circ} \mathrm{C}$ após a coleta. A concentração total de proteínas no humor aquoso foi determinada por meio de um teste colorimétrico. Resultados: A média geométrica da concentração total de proteínas no humor aquoso foi de $32 \mathrm{mg} / \mathrm{dL}$ (amplitude: 8-137 mg/dL) no grupo glaucoma primário de ângulo aberto e de $16 \mathrm{mg} / \mathrm{dL}$ (amplitude: $2-85 \mathrm{mg} / \mathrm{dL}$ ) no grupo controle. A razão da concentração total de proteínas no humor aquoso entre estes dois grupos foi de 2,0 (intervalo de confiança de 95\%: 1,3 a 3,2; $\mathrm{p}=0,003$ ). Conclusões: A concentração total de proteínas no humor aquoso de pacientes com glaucoma primário de ângulo aberto foi aproximadamente duas vezes maior quando comparada aos pacientes sem glaucoma.

Descritores: Glaucoma de ângulo aberto; Proteínas; Humor aquoso; Catarata; Pressão intra-ocular; Estudo comparativo

\section{INTRODUÇÃOO}

O glaucoma primário de ângulo aberto (GPAA) é a forma mais comum de glaucoma. Projeções recentes estimam que, no mundo todo, 60,5 milhões de pessoas apresentarão alguma forma de glaucoma em 2010, sendo o GPAA responsável por $74 \%$ dos $\operatorname{casos}^{(1)}$. Apesar de tratar-se de doença multifatorial $^{(2)}$, o principal fator de risco para glaucoma é a pressão intra-ocular (PIO) elevada $^{(3-4)}$.

Esta elevação da PIO seria secundária ao aumento da resistência à drenagem do humor aquoso através da malha trabecular, principalmente em sua porção justacanalicular ${ }^{(5)}$. As causas do aumento de resistência na malha trabecular ainda não são bem determinadas. No entanto, é provável que alterações bioquímicas, tais como, diferenças na concentração de ácido hialurônico ${ }^{(6)}$ e ácido ascórbico ${ }^{(7)}$, além de proteínas, como fator transformador de crescimento beta- $2^{(8-9)}$, endotelina ${ }^{(10)}$, cochilina ${ }^{(11)}$ e transferrina ${ }^{(12)}$, 
presentes no humor aquoso e na malha trabecular de pacientes com glaucoma, estejam envolvidas na patogênese da doença.

Em relação à concentração total de proteínas no humor aquoso, em determinado estudo, avaliaram pacientes com GPAA, síndrome de pseudo-esfoliação e catarata ${ }^{(13-14)}$. Os pacientes com pseudo-esfoliação e GPAA apresentaram valores mais elevados de proteínas no humor aquoso quando comparados ao grupo controle. Em diferentes trabalhos de um mesmo grupo, foram descritos depósitos da proteína cochilina na malha trabecular de pacientes com GPAA ${ }^{(11-15)}$. Esta proteína, inicialmente descrita em pacientes com surdez neurosensorial, já havia sido observada pelo mesmo grupo de pesquisadores depositada na malha trabecular de camundongos com glaucoma induzido ${ }^{(15)}$.

Em nosso meio, são escassas as publicações que tratam do conteúdo protéico do humor aquoso de pacientes com GPAA $^{(16-17)}$. Nestes estudos, comparando-se pacientes com GPAA e catarata, não foram encontradas diferenças significativas entre os grupos, contrariando resultados publicados na literatura internacional. Diante disto, este estudo foi elaborado para comparar a concentração total de proteínas no humor aquoso entre pacientes com GPAA e indivíduos sem glaucoma em nosso meio.

\section{MÉTODOS}

O protocolo deste estudo prospectivo foi aprovado pelo comitê de ética da Universidade Federal de São Paulo. Foi obtido consentimento livre e esclarecido de cada paciente envolvido. O estudo foi desenvolvido seguindo as diretrizes da Resolução 196/96 e da Declaração de Helsinque.

\section{Pacientes}

Foram incluídos neste estudo 22 pacientes (22 olhos) com GPAA não controlados clinicamente e 22 pacientes ( 22 olhos) sem glaucoma. Os pacientes do grupo GPAA seguiram os seguintes critérios de inclusão: PIO acima da pressão alvo mesmo com o uso de medicação antiglaucomatosa máxima, alterações do disco óptico sugestivas de glaucoma, defeito de campo visual compatível com dano glaucomatoso, gonioscopia apresentando ângulo aberto 360 graus sem alterações patológicas e ausência de qualquer outra patologia ocular associada significante. As alterações do disco óptico consideradas sugestivas de glaucoma foram: escavação vertical maior que 0,5 , assimetria de escavação entre os olhos maior que 0,2 , defeito localizado da rima neural ou hemorragia peripapilar. As alterações de campo visual (Humphrey SITA 24-2, Carl Zeiss Meditec, Dublin, CA, EUA) consideradas sugestivas de glaucoma foram: defeito perimétrico formado por três ou mais pontos adjacentes não periféricos com $\mathrm{p}<5 \%$ no gráfico do "pattern deviation" ou índice "pattern standard deviation" (PSD) $<5 \%$ ou "glaucoma hemifield test" (GHT) fora dos limites normais.

O grupo controle foi composto de pacientes apresentando catarata senil com PIO menor que $21 \mathrm{mmHg}$, aparência normal do disco óptico e ausência de outras alterações oculares significativas.

\section{Coleta e análise das amostras}

Foram coletadas amostras de humor aquoso de 22 pacientes com GPAA sem controle medicamentoso logo no início da trabeculectomia (grupo GPAA). Na coleta foi aspirado $0,1 \mathrm{~mL}$ de humor aquoso da câmara anterior através de uma agulha de calibre ("gauge") 26. O mesmo procedimento foi realizado com 22 pacientes sem glaucoma logo no início da realização da cirurgia de catarata (grupo controle). A amostra de humor aquoso foi armazenada a $-20^{\circ} \mathrm{C}$ imediatamente após a coleta.

A concentração total de proteínas no humor aquoso foi feita por meio de um teste colorimétrico de acordo com as especificações do fornecedor (Protein Assay Kit - Bio-Rad Laboratories, CA,USA). Neste teste, 20 microlitros de cada amostra de humor aquoso (diluído 1:10 em água destilada) bem como uma curva padrão (0 a $40 \mathrm{mg} / \mathrm{dL}$ ) de albumina bovina (Sigma, Tóquio, Japão) foram aplicados em placas "multiwell" do tipo ELISA (Immunoplate polysorp, Nunc, Roskilde, Dinamarca), em duplicatas. Em cada "well”, contendo as amostras e a curva padrão, foram adicionados 200 microlitros do reagente colorimétrico e, a seguir, a absorbância foi determinada, a 630 nanômetros, em espectrofotômetro ELX800 (Bio-Tek Instruments, Winooski, VT, EUA). A quantificação do total de proteínas (em $\mathrm{mg} / \mathrm{dL}$ ) foi feita comparando-se a absorbância obtida em cada amostra com a absorbância da curva padrão. Nossa opção por esse método se baseou em sua eficiência e praticidade, principalmente quando comparado aos utilizados em estudos anteriores. Na técnica de eletroforese em gel de Laemli, por exemplo, são necessários dois dias para a quantificação do total de proteínas (um dia para o preparo do gel e mais um dia para a eletroforese e densitometria), enquanto no método colorimétrico, toda a análise pode ser feita em menos de duas horas.

O teste $t$ de Student para amostras independentes foi utilizado na comparação entre os grupos com relação à idade e à concentração total de proteínas (após transformação logarítmica devido à distribuição assimétrica positiva) no humor aquoso. Foi adotado o nível de significância estatística de 0,05.

\section{RESULTADOS}

As médias (desvios-padrão) de idade nos grupos GPAA e controle foram, respectivamente, 64,6 anos $(9,7)$ e 67,7 anos $(8,3)(p=0,27)$. A proporção de pacientes do sexo feminino em relação ao masculino foi de 1,4:1 (13 mulheres / 9 homens) no grupo GPAA e de 2,1:1 (15 mulheres / 7 homens) no grupo controle. Nenhum paciente avaliado havia sido submetido a cirurgia ocular prévia. No grupo GPAA, todos os pacientes apresentavam razão linear escavação/disco maior ou igual a 0,8 e estavam utilizando uma combinação de duas ou mais medicações antiglaucomatosas antes da cirurgia (Tabela 1). Todos os pacientes deste grupo estavam em uso de maleato 


\begin{tabular}{|c|c|c|c|c|}
\hline Paciente & Sexo & $\begin{array}{l}\text { Idade } \\
\text { (anos) }\end{array}$ & $\begin{array}{c}\text { Razão } \\
\text { linear vertical } \\
\text { escavação/disco }\end{array}$ & Medicação \\
\hline 1 & Feminino & 77 & 1,0 & $\mathrm{Ti}+\mathrm{Br}+\mathrm{Tr}$ \\
\hline 2 & Feminino & 65 & 1,0 & $\mathrm{Ti}+\mathrm{Tr}$ \\
\hline 3 & Feminino & 71 & 0,9 & $\mathrm{Ti}+\mathrm{Do}+\mathrm{Br}$ \\
\hline 4 & Feminino & 65 & 0,8 & $\mathrm{Ti}+\mathrm{Br}+\mathrm{Bi}$ \\
\hline 5 & Feminino & 64 & 0,9 & $\mathrm{Ti}+\mathrm{Br}+\mathrm{Bi}$ \\
\hline 6 & Masculino & 69 & 1,0 & Ti+La \\
\hline 7 & Masculino & 76 & 1,0 & $\mathrm{Ti}+\mathrm{Tr}$ \\
\hline 8 & Masculino & 73 & 0,8 & $\mathrm{Ti}+\mathrm{Tr}$ \\
\hline 9 & Feminino & 40 & 1,0 & $\mathrm{Ti}+\mathrm{Tr}$ \\
\hline 10 & Masculino & 42 & 0,9 & $\mathrm{Ti}+\mathrm{Bz}$ \\
\hline 11 & Masculino & 70 & 0,9 & $\mathrm{Ti}+\mathrm{Br}$ \\
\hline 12 & Masculino & 67 & 1,0 & $\mathrm{Ti}+\mathrm{Br}+\mathrm{Tr}$ \\
\hline 13 & Feminino & 61 & 1,0 & $\mathrm{Ti}+\mathrm{Br}+\mathrm{Tr}$ \\
\hline 14 & Feminino & 63 & 0,9 & $\mathrm{Ti}+\mathrm{Do}+\mathrm{Tr}$ \\
\hline 15 & Masculino & 57 & 1,0 & $\mathrm{Ti}+\mathrm{Do}+\mathrm{Tr}$ \\
\hline 16 & Feminino & 60 & 0,9 & $\mathrm{Ti}+\mathrm{Do}+\mathrm{Tr}$ \\
\hline 17 & Feminino & 58 & 0,9 & $\mathrm{Ti}+\mathrm{Br}$ \\
\hline 18 & Feminino & 70 & 1,0 & $\mathrm{Ti}+\mathrm{Br}+\mathrm{La}$ \\
\hline 19 & Feminino & 64 & 1,0 & $\mathrm{Ti}+\mathrm{Tr}$ \\
\hline 20 & Feminino & 62 & 1,0 & $\mathrm{Ti}+\mathrm{Br}+\mathrm{Tr}$ \\
\hline 21 & Masculino & 79 & 1,0 & $\mathrm{Ti}+\mathrm{Br}+\mathrm{Tr}$ \\
\hline 22 & Masculino & 70 & 1,0 & $\mathrm{Ti}+\mathrm{Br}+\mathrm{Bi}$ \\
\hline \multicolumn{5}{|c|}{$\begin{array}{l}\mathrm{Ti}=\text { maleato de timolol } 0,5 \% ; \mathrm{Br}=\text { tartarato de brimonidina } 0,2 \% ; \mathrm{Tr}=\text { travaprosta } \\
0,004 \% ; \mathrm{Do}=\text { dorzolamida } 2 \% ; \mathrm{Bz}=\text { brinzolamida } 1 \% ; \mathrm{Bi}=\text { bimatoprosta } 0,03 \% \\
\mathrm{La}=\mathrm{Latanoprosta} 0,005 \% .\end{array}$} \\
\hline
\end{tabular}

de timolol 0,5\%. Em 18 pacientes (82\%), análogos de prostaglandina ou prostamida estavam sendo administrados. Em relação às outras medicações, 12 pacientes $(55 \%)$ estavam em uso de alfa-agonistas e 5 pacientes $(23 \%)$ utilizavam inibidores tópicos da anidrase carbônica.

A média geométrica da concentração total de proteínas no humor aquoso foi de $32 \mathrm{mg} / \mathrm{dL}$ (amplitude: $8-137 \mathrm{mg} / \mathrm{dL}$ ) no grupo GPAA e de $16 \mathrm{mg} / \mathrm{dL}$ (amplitude: $2-85 \mathrm{mg} / \mathrm{dL}$ ) no grupo controle. A razão da concentração total de proteínas no humor aquoso entre os grupos foi de 2,0 (intervalo de confiança de $95 \%: 1,3$ a 3,$2 ; \mathrm{p}=0,003)$.

\section{DISCUSSÃO}

Neste estudo, observou-se que a concentração total de proteínas no humor aquoso de pacientes com GPAA foi o dobro da encontrada em pacientes sem glaucoma. Estes resultados são concordantes com os encontrados na literatura mundial $^{(9-10,12)}$. Em determinado estudo, no qual foram avaliados 36 pacientes com GPAA e 33 sem glaucoma, encontrou-se uma diferença ainda maior entre os grupos, com valores de $62,11 \mathrm{mg} / \mathrm{dL}$ e $11,87 \mathrm{mg} / \mathrm{dL}$, respectivamente ${ }^{(12)}$.

Por outro lado, dois estudos publicados na literatura nacional não observaram diferenças entre pacientes com e sem glaucoma. No primeiro estudo, foram encontrados valores de
19,35 mg/dL em pacientes com GPAA e 20,54 mg/dL em indivíduos sem glaucoma ${ }^{(16)}$. No segundo, em um estudo que avaliou tanto pacientes com glaucoma associado à pseudo-esfoliação quanto com GPAA, foram observados concentrações de 24,69 mg/dL em indivíduos com GPAA e de 21,88 mg/dL em pacientes sem glaucoma ${ }^{(17)}$. Esta diferença em relação aos resultados que encontramos talvez possa ser explicada por duas razões: uso de medicação antiglaucomatosa e método de dosagem de proteína.

Os pacientes com GPAA, nos dois estudos supracitados, não estavam em uso de medicação antiglaucomatosa ou tiveram o tratamento suspenso 48 horas antes da cirurgia. Em nosso estudo, todos os pacientes com glaucoma estavam em uso de pelo menos duas medicações e não houve interrupção do tratamento antes da cirurgia.

Em relação à análise quantitativa de proteínas no humor aquoso, o método utilizado no primeiro estudo ${ }^{(16)}$ foi a técnica de eletroforese em gel de Laemli, enquanto que em nosso estudo optamos pela análise por meio de um teste colorimétrico.

Estudos na literatura demonstraram aumento da concentração total de proteínas em pacientes com GPAA $^{(9-10,12)}$ e associaram esta alteração a dois mecanismos principais: quebra de barreira hemato-aquosa ${ }^{(18)}$ e uso de medicação antiglaucomatosa $\mathrm{a}^{(19-20)}$.

O mecanismo de quebra de barreira tem sido implicado como o principal responsável pelo aumento de proteínas no humor aquoso de pacientes com $\mathrm{GPAA}^{(18,21)}$. Este mecanismo pôde ser demonstrado através de estudos com angiofluoresceinografia e iridocromoscopia iriana ${ }^{(21-22)}$. Foram observadas na angiofluoresceinografia alterações da permeabilidade vascular iriana, secundárias à hipoperfusão e microneovascularização no círculo arterial menor e na região ciliar.

Em relação ao uso de medicações antiglaucomatosas, estudos prévios demonstraram aumento na concentração total de proteínas no humor aquoso com o uso tópico de maleato de timolol 0,5\%. Provavelmente este aumento de concentração se deve a uma diminuição da ultrafiltração ${ }^{(19-20)}$. Em um estudo onde se comparou latanoprosta em diferentes concentrações e maleato de timolol $0,5 \%$, foi demonstrado que apenas o timolol estava relacionado ao aumento na concentração total de proteínas no humor aquoso ${ }^{(23)}$. Todos os pacientes em nosso grupo de GPAA estavam utilizando maleato de timolol 0,5\%, o que poderia ser uma explicação para a maior concentração de proteínas nesse grupo. Quanto ao efeito de outras medicações antiglaucomatosas, foi descrito um aumento na concentração total de proteínas no humor aquoso após administração oral de $500 \mathrm{mg}$ de acetazolamida ${ }^{(24)}$. Após extensa revisão da literatura, não encontramos relatos sobre a influência do uso de inibidores tópicos da anidrase carbônica ou de alfa-agonistas na concentração total de proteínas no humor aquoso.

O grupo controle ideal seria composto por pacientes sem alterações oculares. Entretanto, seria antiético colhermos humor aquoso de pacientes sem indicação cirúrgica. Desta forma, optamos por colher de pacientes que seriam submetidos à cirurgia de catarata. Em relação à influência da catarata sobre a concentração protéica do humor aquoso, foi demonstrado ante- 
riormente não haver diferenças entre a concentração total de proteínas de pacientes com catarata, pseudofácicos e afáci$\cos ^{(25)}$. Entretanto, nos casos de glaucoma facolítico, ocorre um aumento da concentração de proteína no humor aquoso ${ }^{(26)}$. No entanto, nenhum paciente do grupo controle do presente estudo apresentava-se com glaucoma facolítico. Diante disto, acreditamos que a concentração de proteínas no humor aquoso em pacientes com GPAA em tratamento clínico seja maior do que em indivíduos não-glaucomatosos portadores de catarata senil.

\section{CONCLUSÕES}

No presente estudo, a concentração total de proteínas no humor aquoso de pacientes com GPAA em tratamento clínico foi duas vezes superior em relação a indivíduos não glaucomatosos portadores de catarata senil. Futuros estudos se fazem necessários para elucidar o real efeito das drogas antiglaucomatosas neste sentido e o eventual papel deste achado na fisiopatologia do glaucoma, caso ocorra mesmo na ausência do uso de drogas.

\section{ABSTRACT}

Purpose: To compare total protein concentration in the aqueous humor of primary open-angle glaucoma and non-glaucomatous patients. Methods: Aqueous humor samples were obtained from 22 patients just before trabeculectomy for clinically uncontrolled primary open angle glaucoma (POAG group). Aqueous humor $(0.1 \mathrm{~mL})$ was aspirated by inserting a 26-gauge needle into the anterior chamber. The same procedure was performed in 22 non-glaucomatous patients just before cataract surgery (control group). Immediately after collection, the aqueous humor was stored at $-20^{\circ} \mathrm{C}$. Aqueous humor total protein concentration was determined using a colorimetric assay. Results: The geometric mean of total protein concentration of the aqueous humor samples was $32 \mathrm{mg} / \mathrm{dL}$ (range: $8-137 \mathrm{mg} / \mathrm{dL}$ ) in the primary open angle glaucoma group and $16 \mathrm{mg} / \mathrm{dL}$ (range: $2-85 \mathrm{mg} / \mathrm{dL}$ ) in the control group. The ratio of the protein concentration between the two groups was $2.0(95 \%$ confidence interval: 1.3 to $3.2 ; \mathrm{p}=0.003)$. Conclusions: The total protein concentration in primary open-angle glaucoma aqueous humor was approximately two times higher than that in nonglaucomatous subjects.

Keywords: Glaucoma, open-angle; Proteins; Aqueous humor; Cataract; Intraocular pressure; Comparative study

\section{REFERÊNCIAS}

1. Quigley HA, Broman AT. The number of people with glaucoma worldwide in 2010 and 2020. Br J Ophthalmol. 2006;90(3):262-7. Comment in: Br J Ophthalmol. 2006;90(3):253-4.

2. Flammer J, Orgul S. Optic nerve blood-flow abnormalities in glaucoma. Prog Retin Eye Res. 1998;17(2):267-89.
3. Evans DW, Hosking SL, Gherghel D, Bartlett JD. Contrast sensitivity improves after brimonidine therapy in primary open angle glaucoma: a case for neuroprotection. Br J Ophthalmol. 2003;87(12):1463-5.

4. Gordon MO, Beiser JA, Brandt JD, Heuer DK, Higginbotham EJ, Johnson CA, et al. The Ocular Hypertension Treatment Study: baseline factors that predict the onset of primary open-angle glaucoma. Arch Ophthalmol. 2002; 120(6):714-20; discussion 829-30. Comment in: Arch Ophthalmol. 2004;122 (7):1088-9; author reply 1089 .

5. Maepea O, Bill A. Pressures in the juxtacanalicular tissue and Schlemm's canal in monkeys. Exp Eye Res. 1992;54(6):879-83.

6. Navajas EV, Martins JRM, Melo LAS Jr, Saraiva VS, Dietrich CPV, Nader $\mathrm{HB}$, et al. Concentration of hyaluronic acid in primary open-angle glaucoma aqueous humor. Exp Eye Res. 2005;80(6):853-7.

7. Koliakos GG, Konstas AG, Schlotzer-Schrehardt U, Bufidis T, Georgiadis N, Ringvold A. Ascorbic acid concentration is reduced in the aqueous humor of patients with exfoliation syndrome. Am J Ophthalmol. 2002;134(6):879-83.

8. Fawthrop FW, Frazer A, Russel RG, Bunning RA. Effects of transforming growth factor beta on the production of prostaglandin $\mathrm{E}$ and caseinase activity of unstimulated and interleukin 1-stimulated human articular chondrocytes in culture. Br J Rheumatology. 1997;36(7):729-34.

9. Picht G, Welge-Luessen U, Grehn F, Lutjen-Drecoll E. Transforming growth factor beta 2 levels in the aqueous humor in different types of glaucoma and the relation to filtering bleb development. Graefes Arch Clin Exp Ophthalmol. 2001;239(3):199-207.

10. Koliakos GG, Konstas AGP, Schlotzer-Schrehardt U, Hollo G, Mitova D, Kovatchev D, et al. Endothelin-1 concentration is increased in the aqueous humour of patients with exfoliation syndrome. Br J Ophthalmol. 2004;88(4): 523-7.

11. Bhattacharya SK, Rockwood EJ, Smith SD, Bonilha VL, Crabb JS, Kuchtey $\mathrm{RW}$, et al. Proteomics reveal cochlin deposits associated with glaucomatous trabecular meshwork. J Biol Chem. 2005;280(7):6080-4.

12. Tripathi RC, Borisuth NS, Tripathi BJ, Gotsis SS. Quantitative and qualitative analyses of transferrin in aqueous humor from patients with primary and secondary glaucomas. Invest Ophthalmol Vis Sci. 1992;33(10):2866-73.

13. Kuchle M, Ho TS, Nguyen NX, Hannappel E, Naumann GO. Protein quantification and electrophoresis in aqueous humor of pseudoexfoliation eyes. Invest Ophthalmol Vis Sci. 1994;35(2):748-52.

14. Kuchle M, Nguyen NX, Hannappel E, Beck W, Ho ST, Naumann GO. Tyndallometry with the laser flare cell meter and biochemical protein determination in the aqueous humor of eyes with pseudoexfoliation syndrome. Ophthalmologe. 1994;91(5):578-84.

15. Bhattacharya SK, Annangudi SP, Salomon RG, Kuchtey RW, Peachey NS, Crabb JW. Cochlin deposits in the trabecular meshwork of the glaucomatous DBA/2J mouse. Exp Eye Res. 2005;80(5):741-4.

16. Almeida GV, Camargo LR, Cohen R, Belfort R. Conteúdo protéico do humor aquoso de portadores de glaucoma primário de ângulo aberto. Arq Bras Oftalmol. 1996;59(2):151-5.

17. Cohen R, Lopes JD, Gesztesi JL, Moraes JZ, Almeida GV, Moreira JBC. Análise quantitativa e qualitativa do conteúdo protéico do humor aquoso de portadores de síndrome de exfoliação. Arq Bras Oftalmol. 1996;59(3):280-4.

18. Kuryshova NI, Vinetskaia MI, Erichev VP, Artamonov VP, Uspenskaia AP. Permeability of blood-aqueous humor barrier in primary open-angle glaucoma. Vestn Oftalmol. 1998;114(1):10-3.

19. Beardsley TL, Shields MB. Effect of timolol on aqueous humor protein concentration in humans. Am J Ophthalmol. 1983;95(4):448-50.

20. Stur M, Grabner G, Huber-Spitzy V, Schreiner J, Haddad R. Effect of timolol on aqueous humor protein concentration in the human eye. Arch Ophthalmol. 1986;104(6):899-900.

21. Vodovozov AM, Rybnikov AA. Iris examination in transformed light in primary open-angle glaucoma. Vestn Oftalmol. 1991;107(1):31-6.

22. Wodowozow AM, Rybnikov AA. Comprehensive study of the iris in transformed light in simple glaucoma. Klin Monatsbl Augenheilkd. 1991;199(4): 246-50.

23. Diestelhorst M, Roters S, Krieglstein GK. The effect of latanoprost $0.005 \%$ once daily versus $0.0015 \%$ twice daily on intraocular pressure and aqueous humour protein concentration in glaucoma patients. A randomized, doublemasked comparison with timolol 0.5\%. Graefes Arch Clin Exp Ophthalmol. 1997;235(1):20-6.

24. Oshika T, Araie M. Time course of changes in aqueous protein concentration and flow rate after oral acetazolamide. Invest Ophthalmol Vis Sci. 1990;31(3): 527-34.

25. Tuberville AW, Wood TO. Aqueous humor protein and complement in pseudophakic eyes. Cornea. 1990;(3):249-53.

26. Silva MRBM, Parente DR, Schellini SA, Jorge EM, Jorge AH, Gaiotto PC. Glaucoma facolítico: correlação clínica com proteínas no humor aquoso. Arq bras Oftalmol. 1996;59(1):31-6. 